\title{
Avaliação dos estressores no trânsito: desenvolvimento da escala de estressores trânsito (ESET)
}

\section{Evaluation of transit stressors at: development of escala de estressores do trânsito (ESET)}

\author{
Marcelo Mendes dos Santos* \\ Universidade do Sagrado Coração - USC, Bauru, São Paulo, Brasil
}

\author{
Hugo Ferrari Cardoso** \\ Universidade do Sagrado Coração - USC, Bauru, São Paulo, Brasil
}

Thelma Margarida de Moraes dos Santos***

Universidade do Sagrado Coração - USC, Bauru, São Paulo, Brasil

\begin{abstract}
RESUMO
A presente pesquisa teve como objetivos construir e buscar evidências de validade e precisão de uma medida psicológica denominada Escala de Estressores do Trânsito (ESET). Participaram deste estudo 472 sujeitos, os quais responderam à escala, inicialmente composta por 72 itens. Os resultados alcançados após análise fatorial permitiram evidenciar validade de construto, permanecendo 37 itens, explicando $62,17 \%$ da variância, com as cargas fatoriais acima de 0,40 e confiabilidade de 0,96. A ESET apresentou quatro fatores: Veículo (19 itens com $a=0,96)$; Condutor (sete itens e com $a=0,87$ ); Vias (sete itens e com $a=0,80$ ) e Ambiente (quatro itens e $a=0,83$ ). Discute-se acerca da importância de construção de instrumentos voltados para a área da Psicologia do Trânsito, haja vista a escassez de instrumentos que mensurem estressores para o contexto do trânsito brasileiro.
\end{abstract}

Palavras-chave: Avaliação psicológica; Construção de instrumento psicológico; Estresse; Psicologia do trânsito.

\footnotetext{
ABSTRACT

The This study aimed to build and provide evidence of validity and accuracy of a psychological measure called Escala de Estressores do Trânsito (ESET). The study included 472 subjects who responded to the scale originally comprised 72 items. The result after factor analysis enabled us to construct validity remaining 37 items, explaining $62.17 \%$ of the variance, with factor loadings above 0.40 and reliability of 0.96 . The ESET presented four factors: vehicle (19 items with $a=0.96)$; Driver (seven items and with $a=0.87$ ); Routes (seven items and with $a=0.80$ ) and Environment (four items and $a$ $=0.83$ ). It discusses about the importance of building instruments focused on the area of Traffic Psychology, considering the scarcity of instruments that measure stressors to the context of the Brazilian traffic.
} 
Keywords: Psychological evaluation; Construction of a psychological tool; Stress; Traffic psychology.

\section{Introdução}

A construção de um instrumento psicológico é um processo que demanda tempo, além de não ser um procedimento simples, uma vez que perpassa por várias etapas para a consecução desse objetivo. Por outro lado, mesmo resguardando toda dificuldade no processo de construção, os pesquisadores devem persistir na realização destes estudos, disponibilizando à comunidade científica melhores instrumentos que ofereçam dados confiáveis e bons processos de avaliação psicológica (NORONHA; FREITAS; OTTATI, 2002).

A respeito dos instrumentos psicológicos, de acordo com Cronbach (1996), Casullo (1999) e Anastasi e Urbina (2000), um teste psicológico é uma medida objetiva e padronizada de uma amostra de comportamento, cujo objetivo é comparar os comportamentos de sujeitos. É um procedimento sistemático para observar o comportamento e descrevê-lo. Para Cruz (2007), os testes psicológicos exercem a função de conhecimento de fenômenos psicológicos dentro de um processo de avaliação. A aplicação de instrumentos é de grande auxílio por proporcionar a inferência de algumas constatações.

De acordo com Pasquali (1999a):

De um segundo plano desprestigiado, a avaliação psicológica vem se tornando ultimamente uma panaceia para resolução de qualquer problema humano, na educação, na saúde, na orientação vocacional, na seleção e qualificação profissional, em tudo. [...] Hoje em dia, além dessa preocupação, outro objetivo vem assumindo proporções agigantadas dentro da avaliação psicológica. [...] Uma característica marcante e crescente desta tendência pode ser visualizada nas tentativas, também crescentes, de impor a obrigatoriedade da avaliação psicológica via legislação, tornando, inclusive, criminalmente responsável o profissional dessa área (p. 21).

A Psicologia do Trânsito vem mostrando sua importância nos últimos tempos, em razão dos diversos âmbitos de atuação, seja pelo estudo e mensuração de comportamentos individuais e sociais dos indivíduos, contribuindo para melhorar as situações das estradas, sinalizações ou até mesmo em práticas de educação para o trânsito (ROZESTRATEN, 1988; SILVA; GÜNTHER, 2005).

Por trânsito, Rozestraten (1988) concebe como o deslocamento pelas vias de veículos e pedestres que seguem a normas e procedimentos a fim de manter a integridade dos mesmos. Porém a realidade tem mostrado que o crescimento da frota de veículos vem ameaçando 
cada vez mais a integridade das pessoas, requerendo medidas mais efetivas, visto que este fato pode causar mais acidentes, congestionamentos e, por conseguinte, estresse nos condutores (AYRES; FERRI, 2004; CAMPOS, 2005; SILVA; GÜNTHER, 2005).

No que se refere ao conceito de estresse, Lipp e Malagris (2001) destacam que qualquer evento que proporcione uma emoção ao indivíduo, seja ela boa ou má, pode ser considerada fonte de estresse. Tais autoras salientam também a importância dos fatores estressores, ou seja, um evento é considerado causador de estresse quando percebido pelo indivíduo dessa forma. Em acréscimo, existem dois tipos de estresse: o considerado positivo (eustress); e o negativo (distress). O primeiro ocorre na medida em que as emoções boas levam à quebra da homeostase interna do indivíduo. Entretanto, nesses casos o estresse tende a diminuir mais rapidamente, já que pode ser visto como motivador e produtivo ao indivíduo. Ao contrário, no caso do estresse negativo (distress), as cognições concebidas como ruins e geradoras do estresse tendem a se manter por um período maior, levando o indivíduo a um estágio de exaustão emocional, podendo acarretar em consequências mais graves, como por exemplo, depressão, úlceras gástricas, diabetes, dentre outras.

Acrescentando algumas informações a respeito do estresse, Lipp (2003) ressalta que, o mesmo ocorre em momentos de tensão interna do indivíduo, sendo que este pode acarretar em comprometimentos, tais como: irritabilidade, azia, tensão muscular, insônia ou sonolência e outros. Especificamente no contexto do trânsito, Zanelato e Oliveira (2004) atentam para o fato de que o trânsito pode ser prejudicial à saúde física e psíquica daquele que conduz o veículo, causando, por exemplo, estresse, fadiga, ansiedade, depressão, fobias, doenças cardiovasculares, gastrointestinais, músculo esquelética, dor nas costas e pescoço entre outros.

Nesta mesma direção, de acordo com diferentes autores, o estresse pode influenciar os condutores nas tomadas de decisões no trânsito (ANDRADE, 2007; CARVALHO; MALAGRIS, 2007). Colocar esta frase no parágrafo anterior.

Para Rozestraten (2003) o ato de dirigir é um tanto complexo, pois envolve os comportamentos no trânsito e a convivência com o ambiente social. Por sua vez, a Psicologia tem a possibilidade de estudar e avaliar as características dos condutores de modo sistemático e, por conseguinte, colaborar na elaboração de ações que venham a intervir neste contexto.

Assim, salientamos que o estresse é prejudicial no âmbito do trânsito, em razão de suas consequências que possam vir a desencadear os seus efeitos não apenas no motorista estressado, mas também nos outros condutores. A presente pesquisa se propôs abordar os 
estressores do trânsito por meio da construção de um instrumento psicológico, intitulado Escala de Estressores do Trânsito (ESET).

\section{Objetivos}

Objetivo Geral

- Buscar evidências de validade de construto para a ESET.

Objetivos Específicos

- Realizar análise fatorial exploratória do conjunto de itens construídos

-Excluir itens que não estão de acordo com os parâmetros psicométricos aceitáveis

- Realizar análise fatorial confirmatória do conjunto de itens resultante pós análise fatorial exploratória

- Análise da confiabilidade do instrumento

\section{Método}

Participantes

Participaram do estudo 472 condutores (289 sexo masculino e 172 do sexo feminino e 11 sujeitos que não responderam a essa variável), com idades variando de 18 a 80 anos, distribuídos em 41 cidades do interior do estado de São Paulo. Com relação à carteira de habilitação, 214 eram de categoria $B$, seguidos da categoria D com 107, categoria A com 64, categoria E com 37, categoria C com 36 motoristas, além de 14 que não informaram a categoria da carteira de habilitação. No que se refere à escolaridade dos participantes os níveis que apresentaram maiores freqüências foram Ensino Médio completo ( $n=159)$ e Ensino Superior completo $(n=159)$.

\section{Instrumento}

Escala de Estressores do Trânsito (ESET)

A ESET em sua primeira versão foi composta por 72 itens, construídos por docentes do Programa de Pós-Graduação da Universidade do Sagrado Coração (USC). Os 72 itens foram listados e adaptados para a língua portuguesa no sentido de adequar e evitar palavras pouco utilizadas no cotidiano de nossa língua (normatização linguística). Também foram feitas adaptações para padronização do tipo de pergunta para a escala Likert de cinco pontos (Nunca, Quase nunca, As vezes, Quase sempre, Sempre), além da padronização do tempo verbal das afirmações. Os itens foram alocados em quatro 
dimensões (Veículo, Condutor, Vias e Ambiente) por meio de análise de conteúdo (SANTOS; CARDOSO; SANTOS, 2009).

\section{Procedimentos}

A ESET foi entregue aos alunos dos cursos de Capacitação e Complementação em Perito Examinador do Trânsito e esses foram orientados quanto da sua aplicação bem como solicitados que aplicassem em 10 sujeitos que possuíam habilitação. Antes de responderem ao instrumento os participantes preencheram o Termo de Consentimento Livre e Esclarecido (TCLE), impresso em duas vias, no qual constavam todas as informações pertinentes da pesquisa em questão.

\section{Plano de Análise de dados}

Foi realizada a análise fatorial exploratória da ESET. Entretanto, para que a mesma pudesse ser realizada foi estudada a adequação da amostra pelo determinante da matriz de correlação e pelo teste de Kaiser-Meyer-Olkin (KMO), medida de diagnóstico da homogeneidade dos itens. A extração dos fatores foi realizada por meio do modelo de Análise dos Eixos Principais, que está relacionado com a explicação da estrutura de covariância por meio de combinações lineares dos itens originais, com rotação Promax. O objetivo foi encontrar um meio de condensar a informação contida em um número de itens, em um conjunto menor de variáveis estatísticas com uma perda mínima de informação.

Para determinar o número de fatores para representar a estrutura latente dos dados da escala foi utilizado o critério da raiz latente, o qual seleciona apenas os fatores cujos autovalores são superiores a um, e o teste de Scree que é determinado fazendo-se o gráfico das raízes latentes em relação ao número de fatores em sua ordem de extração, e a forma da curva resultante foi usada para avaliar o ponto de corte. Para análise da confiabilidade dos fatores resultantes, bem como do conjunto de item em sua totalidade, foi utilizado o teste alfa de Cronbach.

\section{Resultados e Discussões}

\subsection{Análise Fatorial Preliminar}

Foi realizada uma análise fatorial exploratória, a fim de se avaliar se os 72 itens eram passíveis de ser fatorados e quantas dimensões 
uma primeira análise sugeriria, utilizando como parâmetros eigenvalues igual ou maior que 1,0 e cargas fatoriais acima de 0,40. Deste modo, o KMO foi de 0,95 com nível de significância de 0,000, apresentando $\chi^{2}=18358,6$. Nessa análise foram retirados 12 fatores, explicando $69,6 \%$ da variância total e o Scree-Plot sugeriu um fator determinante, convertido em nove interações.

\subsection{Análise Fatorial Confirmatória:}

O passo posterior foi retirar os itens que se encontravam em mais de um fator, excluir aqueles que possuíam conteúdos semelhantes, com cargas fatoriais abaixo de 0,40 e eingevalues abaixo de 1,0. O KMO encontrado na segunda análise foi de 0,95 e o teste de esfericidade de Bartlett foi significativo ao nível de 0,000, apresentando $\chi^{2}=10323,4(\mathrm{df}=666)$, demonstrando que as correlações parciais foram bastante satisfatórias para dar prosseguimento ao modelo de análise fatorial. De acordo com Pereira (1999), ao se referir sobre os valores do KMO destaca que, valores acima de 0,80 já é indicio de boa adequação dos dados. A Tabela 1 mostra a variância explicada pelas quatro dimensões encontradas na análise da ESET, contendo 37 itens, explicando $62,17 \%$ da variância total.

A Tabela 1 mostra a variância explicada pelas quatro dimensões encontradas na análise da ESET contendo 37 itens, explicando $62,17 \%$ da variância total.

Tabela 1. Total de Variância Explicada

\begin{tabular}{cccc}
\hline Componentes & Total & Variância (\%) & Variância Acumulada (\%) \\
\hline 1 & 15,813 & 42,738 & 42,738 \\
2 & 3,600 & 9,729 & 52,467 \\
3 & 2,163 & 5,847 & 58,314 \\
4 & 1,428 & 3,859 & 62,174 \\
\hline
\end{tabular}

Método de Extração: Análise dos Eixos Principais.

O Scree-Plot é apresentado na Figura 1 e indicou a preleção de quatro grandes fatores para a escala. Vale ressaltar que o primeiro fator apresentou uma variância bastante superior aos outros três fatores. 
Figura 1. Gráfico de Sedimentação dos Eingevalues.

\section{Scree Plot}

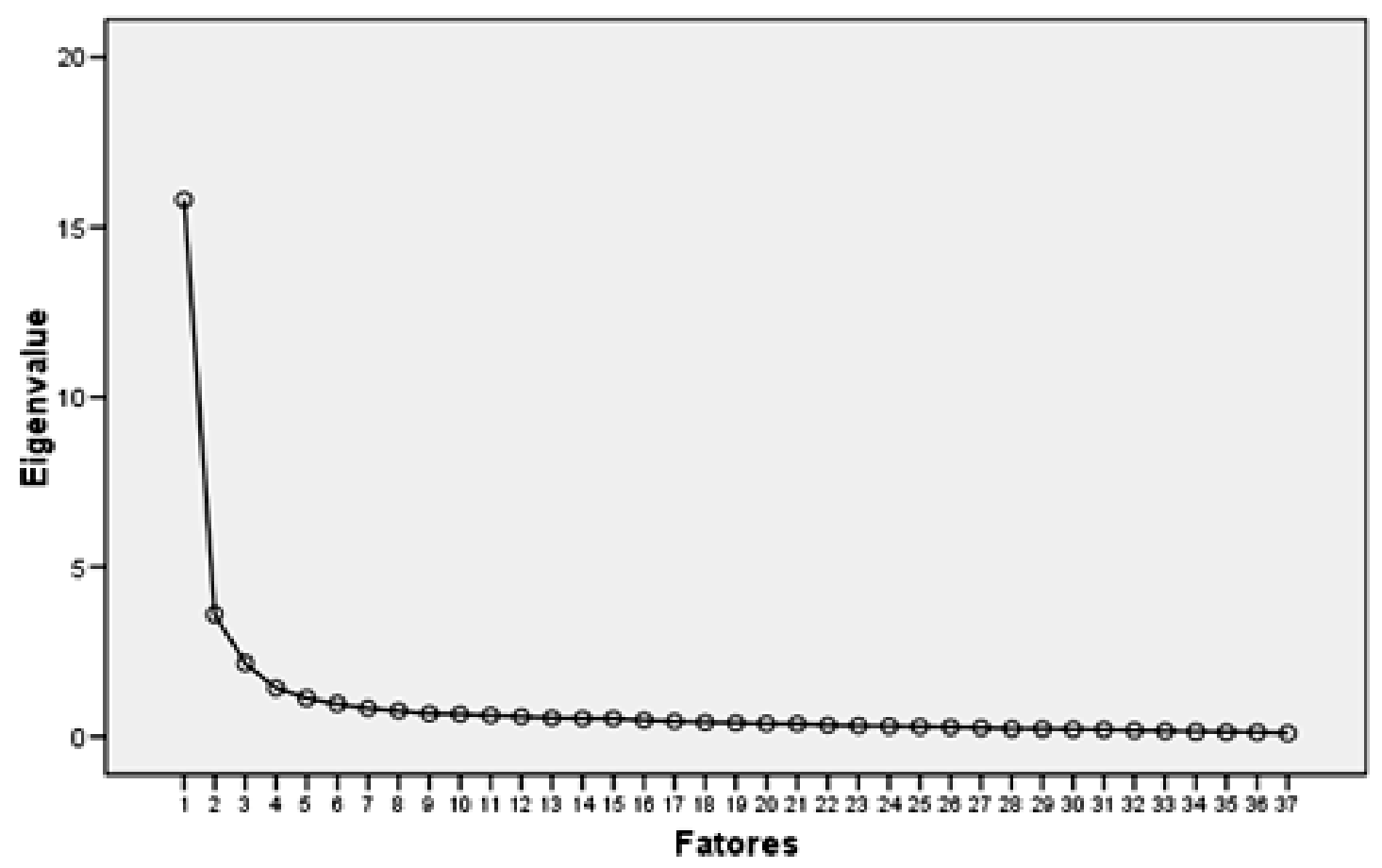

\section{Caracterização e interpretação dos fatores}

Na Tabela 2 são apresentadas as cargas fatoriais do modelo final da ESET convertida em seis interações. Os fatores encontrados receberam as seguintes denominações e composições: Fator 1 (Veículo), composto por 19 itens; o fator 2 (Condutores), composto por sete itens; o fator 3 (Vias), composto por sete itens; o fator 4 (Ambiente), composto por quatro itens.

O fator 1 (Veículo) ficou constituído de itens que estão relacionados a situações em que não há manutenção do meio de transporte, tais como "Falta do retrovisor", "Falta de buzina", "Farol quebrado". O fator 2 (Condutores) aglutinou os itens referentes a ações que outros motoristas realizam que podem ser consideradas estressoras, como por exemplo, "Motoristas que fazem ultrapassagem perigosa". "Motoristas imprudentes", "Motoristas buzinando". No fator 3 (Vias) agruparam-se os itens referentes às características das vias, tais como: "Desvios nas vias", "Presença de lombadas", "Barulho na via". Por último, no fator 4 (Ambiente) aglomeraram-se os itens sobre as condições climáticas que podem ser estressoras, "Neblina", "Vento", "Chuva". 
Tabela 2. Cargas Fatoriais dos Componentes Rotados (Promax) extraídos de Fatores do Eixo Principal e Comunalidades da ESET.

\begin{tabular}{|c|c|c|c|c|c|}
\hline Componentes & 1 & 2 & 3 & 4 & $\mathrm{~h}^{2}$ \\
\hline ESET 62 & 951 & & & & 825 \\
\hline ESET 61 & 941 & & & & 814 \\
\hline ESET 30 & ,901 & & & & 793 \\
\hline ESET 57 & 806 & & & & 788 \\
\hline ESET 63 & 805 & & & & 781 \\
\hline ESET 64 & 799 & & & & 787 \\
\hline ESET 59 & 781 & & & & 744 \\
\hline ESET 31 &, 781 & & & &, 766 \\
\hline ESET 29 &, 779 & & & & 628 \\
\hline ESET 65 &, 760 & & & & ,784 \\
\hline ESET 14 &, 742 & & & & .575 \\
\hline ESET 60 & 739 & & & & 588 \\
\hline ESET 39 &, 739 & & & & 710 \\
\hline ESET 43 & 681 & & & &, 578 \\
\hline ESET 26 & 637 & & & & 628 \\
\hline ESET 10 & 637 & & & & 727 \\
\hline ESET 23 &, 623 & & & & 607 \\
\hline ESET 49 &, 583 & & & & 560 \\
\hline ESET 06 &, 572 & & & &, 598 \\
\hline ESET 41 & & 856 & & & ,668 \\
\hline ESET 25 & &, 792 & & & 579 \\
\hline ESET 48 & & 784 & & & 618 \\
\hline ESET 40 & &, 727 & & & 634 \\
\hline ESET 16 & & 639 & & &, 529 \\
\hline ESET 24 & &, 493 & & & 468 \\
\hline ESET 18 & &, 419 & & &, 529 \\
\hline ESET 35 & & & 680 & &, 510 \\
\hline ESET 32 & & & 616 & & 421 \\
\hline ESET 34 & & &, 598 & & .575 \\
\hline ESET 21 & & &, 529 & & 674 \\
\hline ESET 56 & & &, 527 & & ,415 \\
\hline ESET 20 & & &, 452 & & 657 \\
\hline ESET 17 & & & 448 & & ,404 \\
\hline ESET 53 & & & &, 779 & 702 \\
\hline ESET 54 & & & &, 709 &, 707 \\
\hline ESET 55 & & & &, 596 & 543 \\
\hline ESET 07 & & & &, 538 & 461 \\
\hline Eigenvalues & 15,8 & 3,6 & 2,1 & 1,4 & \\
\hline Variância Explicada & 42,7 & 9,7 & 5,8 & 3,8 & \\
\hline Total de Variância Explicada & \multicolumn{5}{|c|}{62,1} \\
\hline
\end{tabular}

Método de Extração: Fatoração do Eixo Principal. Método de Rotação: Promax com Normalização Kaiser.

Como pode ser visualizado, as cargas fatoriais da ESET se apresentaram acima de 0,40, sendo a que apresentou menor carga foi o item "ciclistas imprudentes" do segundo fator com 0,42. A respeito dos parâmetros mínimos de aceitação, Pasquali (1999) ressalta que 0,30 é considerado carga fatorial mínima aceitável de 
um item, dessa forma, esses dados demonstraram todos os itens da escala estão acima desses parâmetros. A Tabela 3 representa as correlações entre as dimensões da ESET. É importante destacar que de acordo com Dancey e Reidy (2006) as correlações foram classificadas como fracas e moderadas.

Tabela 3: Correlação entre as dimensões da ESET

\begin{tabular}{lllll}
\hline Fatores & 1 & 2 & 3 & 4 \\
\hline 1 & 1,00 & 0,58 & 0,28 & 0,59 \\
2 & 0,58 & 1,00 & 0,36 & 0,51 \\
3 & 0,28 & 0,36 & 1,00 & 0,31 \\
4 & 0,59 & 0,51 & 0,31 & 1,00 \\
\hline
\end{tabular}

A Tabela 4 apresenta a análise de itens por intermédio da correlação item-total, separados por fatores a fim de caracterizar mais uma informação sobre evidências de validade de construto da ESET.

Tabela 4. Correlação Item-total por Fator

\begin{tabular}{cccccccc}
\hline Fator 1 & $r$ & Fator 2 & $r$ & Fator 3 & $r$ & Fator 4 & $r$ \\
\hline ESET 62 &, 84 & ESET 41 &, 75 & ESET 35 &, 53 & ESET 53 &, 77 \\
ESET 61 &, 84 & ESET 25 &, 65 & ESET 32 &, 39 & ESET 54 &, 73 \\
ESET 30 &, 81 & ESET 48 &, 71 & ESET 34 &, 60 & ESET 55 &, 63 \\
ESET 57 &, 84 & ESET 40 &, 66 & ESET 21 &, 61 & ESET 07 &, 53 \\
ESET 63 &, 80 & ESET 16 &, 63 & ESET 56 &, 54 & & \\
ESET 64 &, 78 & ESET 24 &, 56 & ESET 20 &, 57 & & \\
ESET 59 &, 82 & ESET 18 &, 58 & ESET 17 &, 49 & & \\
ESET 31 &, 79 & & & & & & \\
ESET 29 &, 68 & & & & & & \\
ESET 65 &, 79 & & & & & & \\
ESET 14 &, 68 & & & & & & \\
ESET 60 &, 65 & & & & & & \\
ESET 39 &, 79 & & & & & & \\
ESET 43 &, 68 & & & & & & \\
ESET 26 &, 75 & & & & & & \\
ESET 10 &, 75 & & & & & & \\
ESET 23 &, 69 & & & & & & \\
ESET 49 &, 64 & & & & & & \\
ESET 06 &, 65 & & & & & & \\
\hline
\end{tabular}

Os valores de correlação item-total considerando cada fator isolado estão expressos na Tabela 4. Como pode ser visto, o item que apresentou menor coeficiente foi "Barulho na via", com $r=0,49$. De acordo com Guilford e Fruchter (1978) o valor mínimo aceitável para esse tipo de análise é de 0,30, assim sendo, todos os itens da ESET 
se apresentaram acima desta condição, ficando facilitada a interpretação de que todos os itens estão bem alinhados. Nesse mesmo sentido, Pasquali (1999) e Dancey e Reidy (2006) explicam que quanto maiores as cargas fatoriais de itens em um fator, maior será a capacidade desses em mensurar o construto.

A confiabilidade, ou fidedignidade, da escala foi estudada por meio do teste de alfa de Cronbach (a) e esse método, como informa Urbina (2007), refere-se ao procedimento que permite constatar certo grau de confiança entre os instrumentos de medidas quando da aplicação desses em uma amostra ou população. A ESET de acordo com as análises apresentou os seguintes índices: 0 fator 1 (Veículo), composto por 19 itens $(a=0,96)$; o fator 2 (Condutores), composto por sete itens $(a=0,87)$; o fator 3 (Vias), composto por sete itens $(a=0,80)$; o fator 4 (Ambiente), composto por quatro itens $(a=0,83)$, Finalmente, a ESET em sua totalidade ficou composta por 37 itens com satisfatória confiabilidade $(a=0,96)$. Constatando as informações apresentadas, Pasquali (1999) salienta que os coeficientes de precisão são considerados aceitáveis para um teste quando estão acima de 0,70. Em acréscimo, Prieto e Muniz (2000) discorrem que nas análises de confiabilidade por meio de consistência interna, os coeficientes são considerados adequados quando se apresentarem entre 0,70 e 0,79. Acima desses, entre 0,80 e 0,84 são considerados bons e, a partir de 0,85 podem ser considerados como excelentes.

\section{Considerações Finais}

O estudo se propôs a construir uma escala de avaliação dos estressores do trânsito, bem como buscar evidência de construto para a mesma. Diante dos resultados encontrados, percebe-se que esses foram satisfatórios e que o instrumento apresentou qualidades, tais como: sua praticidade, rápida aplicação, administração coletiva e propriedades psicométricas satisfatórias. Foi constatada a evidência de validade de construto da ESET, porém torna-se importante a realização de novas pesquisas no sentido de buscar outras evidências de validade para a escala.

O trabalho realizado deu prioridade à teoria clássica dos testes. Embora fosse possível, não foram analisadas variáveis sócioeconômicas (como sexo, idade, escolaridade, cidade, profissão e categoria da carteira de habilitação), optando-se pela execução da análise fatorial, exclusão de itens e a concretização de evidência de validade de construto. A atual formatação da ESET (37 itens) permite análises mais confiáveis dos resultados.

Este estudo faz parte de um Projeto maior de construção, validação, normatização e submissão da ESET para aprovação do Conselho Federal de Psicologia (CFP), visto que não há instrumentos 
reconhecidos, até o momento, pelo referido Conselho, que possam avaliar o estresse no contexto do trânsito. Portanto, a consecução dos objetivos, da presente pesquisa, permitiram avanços, bem como a possibilidade de continuidade do Projeto inicial.

\section{Referências}

ANASTASI, A.; URBINA, S. Testagem Psicológica. 7 ed. Porto Alegre: Artes Médicas, 2000.

ANDRADE, M. O. P. E. M. Nível de Atenção e Sinais e Sintomas de Estresse em Motoristas Com e/ou Sem Infrações e Acidentes, Biblioteca net. Disponível em: <http://www.tede.ucdb.br/>. Acesso em: 20 dez. 2009.

AYRES, N.; FERRI, C. G. M. L. Considerações para a Educação no Trânsito. Colloquium Humanarum, Presidente Prudente, v. 2, n. 1, p. 67-78, 2004.

CAMPOS, M. M. Uma Análise da Relação entre Acidentes de Tráfego e Variáveis Sociais, Econômicas, Urbanas, e Mobilidade na Cidade do Rio de J aneiro. 2005. 147f. Dissertação (Dissertação de Mestrado) - Universidade Federal do Rio de Janeiro, Rio de Janeiro.

CASULLO, M. M. Evaluácion Psicológica. In: WESCHELER, S. M.; GUZZO, R. S. L. Avaliação Psicológica: Perspectiva Internacional. São Paulo: Casa do Psicólogo, 1999, p. 23-39.

CARVALHO, L., MALAGRIS, L. E. N. Avaliação do Nível de Stress em Profissionais de Saúde. Estudos e Pesquisas em Psicologia, Rio de Janeiro, v. 7, n. 3, 2007.

CRONBACH, L. J. Fundamentos da Testagem Psicológica. Porto Alegre: Artes Médicas, 1996.

CRUZ, R. M. O processo de conhecer em avaliação psicológica. In: CRUZ, R. M.; ALCHIERI, J. C.; SARDÁ-JR, J. J. Avaliação e Medidas Psicológicas: Produção do Conhecimento e da Intervenção Profissional. São Paulo: Casa do Psicólogo, 2007, p.15-26.

DANCEY, C. P.; REIDY, J. Estatística sem matemática para Psicologia usando o SPSS para Windows. 3 ed. Porto Alegre: Artmed, 2006.

GUILFORD, J. P.; FRUCHTER, B. Fundamental statistic in psychology and Education. 6 ed. New York: McGraw-Hill, 1978

LIPP, M. E. N. O que eu tenho é stress? De onde ele vem? In: LIPP, M. E. N. O stress está dentro de você. São Paulo: Editora Contexto, 2003, p. 09-18.

LIPP, M. E. N.; MALAGRIS, L. E. N. O stress emocional e seu tratamento. In: RANGÉ, B. P. Psicoterapias cognitivocomportamentais: um diálogo com a psiquiatria. Porto Alegre: Artmed, 2001, p. 475-490. 
NORONHA, A. P. P.; FREITAS, F. A.; OTTATI, F. Parâmetros psicométricos de testes psicológicos de inteligência. Interação em Psicologia, Curitiba, v. 6, n. 2, p. 195-201, 2002.

PASQUALI, L. Instrumentos psicológicos: Manual prático de elaboração. Brasília: LabPAM \& IBAPP, 1999.

PASQUALI, L. Prefácio. In: WECHSLER, M.; GUZZO, R. S. L. Avaliação Psicológica: Perspectiva Internacional. São Paulo: Casa do Psicólogo, 1999a, p. 21-22.

PEREIRA, J. C. R. Análise de dados qualitativos: estratégias metodológicas para as ciências da saúde, humanas e sociais. São Paulo: EDUSP/FAPESP, 1999.

PRIETO, G.; MUNIZ, J. Un modelo para evaluar la calidad de tests usados na Espanha, 2000. Disponível em: <http://www.cop.es/vernumero.asp?id=41>. Acesso: 20 dez. 2009.

SANTOS, M. M.; CARDOSO, H. F.; SANTOS, T. M. M. Escala de Estressores do Trânsito (ESET). Bauru: Manual técnico não publicado, 2009.

SILVA, A. V.; GÜNTHER, H. Características de Itinerário Urbano e Comportamentos Inadequados de um Motorista de Ônibus. Psicologia: Pesquisa \& Trânsito, Belo Horizonte, v. 1, n. 1, p. 3344, 2005.

ROZestraten, R. J. A. Psicologia do Trânsito: Conceitos e processos básicos. São Paulo: Pedagógica e Universitária LTDA, 1988. ROZESTRATEN, R. J. A. Ambiente, Trânsito e Psicologia. In: HOFFMANN, H. M.; CRUZ, M. R.; ALCHIERI, C. J. Comportamento Humano no Trânsito. São Paulo: Casa do Psicólogo, 2003, p. 3146.

URBINA, S. Fundamentos da testagem psicológica. Porto Alegre: Artmed, 2007.

ZANELATO, L. S.; OLIVEIRA, L. C. Estudar os Comportamentos de Riscos e os Fatores Estressantes Presentes no Cotidiano de Motoristas de Ônibus Urbano, Anais do II Seminário Internacional de Estudos e Pesquisa Qualitativos, Bauru, Universidade Sagrado Coração, 2004, p. 1-7. 


\section{Endereço para correspondência}

Marcelo Mendes dos Santos

Universidade Sagrado Coração, Rua Irmã Arminda 10-50, Jardim Brasil, CEP 17011-160, Bauru - SP, Brasil

Endereço eletrônico: marcelo.santos@usc.br

Hugo Ferrari Cardoso

Universidade Sagrado Coração, Rua Irmã Arminda 10-50, Jardim Brasil, CEP 17011-160, Bauru - SP, Brasil

Endereço eletrônico: hugo.posusc@gmail.com

Thelma Margarida de Moraes dos Santos

Universidade Sagrado Coração, Rua Irmã Arminda 10-50, Jardim Brasil, CEP 17011-160, Bauru - SP, Brasil

Endereço eletrônico: thelma.santos@usc.br

Recebido em: $27 / 04 / 2010$

Reformulado: $12 / 11 / 2010$

Aceito para publicação em: 16/11/2010

Acompanhamento do processo editorial: Ana Maria Lopez Calvo de Feijoo

\section{Notas}

*Professor Integral da Universidade do Sagrado Coração - USC, Bauru/São Paulo/Brasil. Mestre em Educação pela Pontifícia Universidade Católica de Campinas/São Paulo/Brasil.

**Mestre em Avaliação Psicológica pela Universidade São Francisco - Itatiba/São Paulo/Brasil.

****Professora Integral da Universidade do Sagrado Coração - USC, Bauru/São Paulo/Brasil. Mestre em Avaliação Psicológica pela Universidade São Francisco Itatiba/São Paulo/Brasil. 